\title{
Secure Blockchain-Enabled DyMonDS Design
}

\author{
Michelle Lauer \\ mflauer@mit.edu \\ Department of Electrical Engineering \\ and Computer Science, MIT
}

\author{
Rupamathi Jaddivada \\ rjaddiva@mit.edu \\ Laboratory for Information and \\ Decision Systems, MIT
}

\author{
Marija Ilić \\ ilic@mit.edu \\ Laboratory for Information and \\ Decision Systems, MIT
}

\begin{abstract}
In this paper, we propose a secure system design for implementing the minimal-information exchange framework to efficiently provide services; the application of this concept is in the context of electricity services. The information being exchanged is dictated by the Dynamic Monitoring and Decision Systems (DyMonDS) platform, which enables optimal global solutions to be derived even in a largely distributed setting. This capability parallels the increasing number of smart Internet of Things (IoT) devices that allow for a responsive and flexible service. These advancements are aligned in the Secure Blockchain-Enabled DyMonDS design, where a secure communication protocol enables smart embedded devices to communicate with local compute nodes; these compute nodes are connected in a meshed blockchain network, providing information security, integrity, and robustness.
\end{abstract}

\section{CCS CONCEPTS}

- Hardware $\rightarrow$ Electronic design automation;

\section{KEYWORDS}

IoT; Distributed service; Secure electricity service; Dynamic Monitoring and Decision Systems (DyMoNDS); Transactive Energy Management (TEM), Smart Grids, Cybersecurity, Blockchain, ModelBased Computer Platforms, Dynamic Computer Architectures, CyberPhysical Systems

\section{ACM Reference Format:}

Michelle Lauer, Rupamathi Jaddivada, and Marija Ilić. 2019. Secure BlockchainEnabled DyMonDS Design. In INTERNATIONAL CONFERENCE ON OMNILAYER INTELLIGENT SYSTEMS (COINS), May 5-7, 2019, Crete, Greece. ACM, New York, NY, USA, 8 pages. https://doi.org/10.1145/3312614.3312654

\section{INTRODUCTION}

This paper concerns a framework for supporting service oriented systems, such as communications, electricity, heat and other goods. While the entire paper illustrates a Dynamic Monitoring and Decision Systems (DyMonDS) framework in the context of electricity services, the concepts are applicable to all these other service oriented industries and to their bundling. They all are undergoing technological and organizational changes which require replacing

Permission to make digital or hard copies of all or part of this work for personal or classroom use is granted without fee provided that copies are not made or distributed for profit or commercial advantage and that copies bear this notice and the full citation on the first page. Copyrights for components of this work owned by others than ACM must be honored. Abstracting with credit is permitted. To copy otherwise, or republish, to post on servers or to redistribute to lists, requires prior specific permission and/or a fee. Request permissions from permissions@acm.org.

COINS, May 5-7, 2019, Crete, Greece

(C) 2019 Association for Computing Machinery.

ACM ISBN 978-1-4503-6640-3/19/05 . \$ \$15.00

https://doi.org/10.1145/3312614.3312654 traditional top-down utility service with a more interactive participation by all system participants. To avoid potentially major complexity, DyMonDS offers a theoretically-sound approach based on minimal information exchange. Notably, the exchange aligns technical, economic and security objectives. Regarding the changing electric energy industry, the distributed generation is amidst a period of rapid growth and development with rise of new technologies such as large-scale offshore wind farms and solar installations. The resulting increased variability is handled by operating conventional generation resources, which are not designed to be operated for such conditions. This results in excessive wear-and-tear on the hardware and consequently increased wholesale electricity price fluctuations, indicating inefficiencies in grid operations [7]. In such a scenario, the ability to act in a distributed setting to serve local energy needs, coupled with a promise of lower electric service costs is highly desirable. This ability is increasingly enabled by utilizing Distributed Energy Resources (DERs) as smart IoT devices with the ability to communicate directly with the grid. DERs include, but are not limited to, a mix of controllable demand devices such as electric vehicles small backup diesel generators, solar photovoltaics, Heating Ventilating, Air-conditioning and Cooling (HVAC) units. Despite the benefits of remote controlling and monitoring of IoT devices through a smart phone and/or automated learning-enabled activation, their integration into the existing physical power grid, presents both technical and economic challenges. More importantly, such technology opens doors to an increased number of security vulnerabilities [23]. There is a grave urgency to secure the power grid in light of the present socio-political situation.

Shown in Fig. 1 is a representative distribution grid that will be utilized for exposition of concepts throughout this paper. It comprises several neighborhoods with heterogeneous IoT devices as indicated by the legend in the figure. Each of these devices is driven by ambient conditions, consumer needs and the willingness to pay. Additionally, there is a large solar photovoltaic installation, injecting highly variable power injections which makes it increasingly challenging to balance the supply-demand. This is primarily because the end-user uncertainty can not be predicted and communicated to the central operator with minimal delays. Even if this were possible, the uncertainty modeling of the enormous number of IoT devices is not only a computationally intensive task, but also may introduce physical measurement errors and/or numerical inaccuracies. Furthermore, this would require solving a non-linear non-convex problem for system-level balancing, while also meeting often contradicting sub-objectives of stake-holders [4]. Engineering literature has offered a wide range of control techniques to address the associated technical challenges, although most of them are based on questionable assumptions in light of the changing energy systems [9]. However, not many studies have targeted ensuring cybersecurity of power grids. A centralized entity cannot be trusted to 


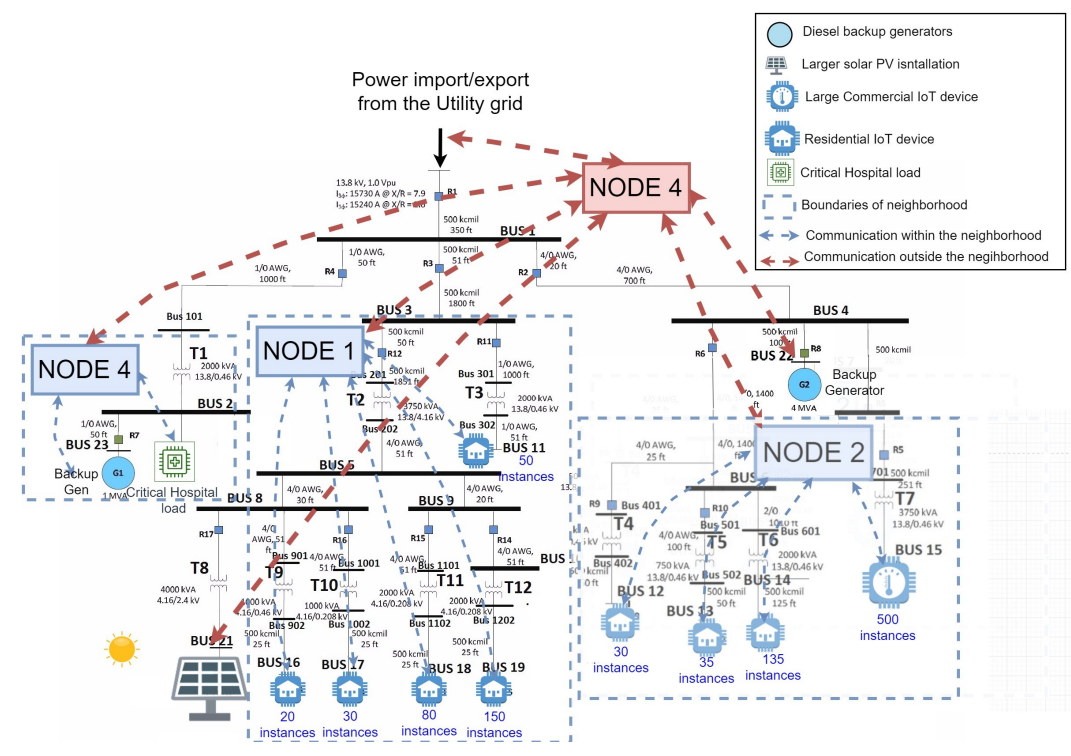

Figure 1: Typical distribution feeder comprising IoT devices communicating with the compute nodes

fully take control of such a grid, because it is generally a single point of failure. At the same time, completely decentralized decision making can never reach the global optimum, resulting in severe market inefficiencies. The success of blockchain technology in addressing these types of challenges in other domains has led researchers to explore how it might be incorporated in energy systems. However, the time criticality and the Quality-of-Service (QoS) requirements, coupled with the various levels of trust between stakeholders of energy systems are hurdles to the application of blockchain in the energy systems. What is desired for overcoming these challenges and adopting blockchain technology is a platform that would allow for minimum information exchange between different entities for preserving security while also having sufficient information to have provable control and accountable market mechanisms.

A generic platform facilitating minimum information exchange between distributed agents is introduced in [8] and is referred to as Dynamic Monitoring and Decision Systems (DyMonDS). The idea behind DyMonDS-enabled platform is to have intelligence embedded in small end-users' smart devices to facilitate the minimum bidirectional information exchange between the end-users and their coordinators, often through multiple layers of hierarchy. Exposing minimal information at interfaces requires rethinking of existing modeling framework. We thus propose to utilize a unified energybased modeling framework introduced in [9] and seek mechanisms to implement DyMonDS in context of providing an integrated solution to the recent technical, economic and cyber challenges the service providers are facing. This energy-based modeling helps decompose otherwise intractable non-linear, non-convex problems into sub-problems allocated to distributed agents, with minimal information exchange between them. This distributed, multi-layered architecture ingrained in the DyMonDS platform demonstrates the ability to arrive at the same optimized results as a fully centralized system. The purpose of this paper is to explore the deployable DyMonDS architecture with energy-based models of decision-makers, with an emphasis on system security throughout the whole process.

We begin with a proposition of how the energy-based modeling can be integrated into the platform design in Section 2. The proposed system design is described in Section 3, including a discussion of how blockchain will be used in the design. Next, Section 4 describes in more detail how various components will be implemented in a secure manner. Finally, in Section 6, we conclude with open questions and ongoing research in the area.

\section{BACKGROUND}

Any physical end-device is governed by constituent dynamics and is driven by external exogenous and controllable cyber inputs [5]. As computational power increases, self-adaptive reinforcementlearning-based controllers have been proposed for these devices. Together, they are expected to form artificial neural networks with self-adaptive and restorative mechanisms for overall system management [16]. However, this is a challenging task due to the human in the loop and market mechanisms overlaid on top of the physical processes. In order for energy systems to function smoothly, operators must take into consideration user preferences at the device level, while also ensuring efficient system-level coordination. We thus propose utilizing energy-based modeling framework for the proposed platform design as will be explained in this section.

Small DERs presently are controlled using ON/OFF signals inbuilt in the analog sensors and relays within. These controllers generally respond to local outputs such as temperature, but not to outside signals such as electricity prices. The fundamental necessity of a device (e.g. solar photo-voltaic, electric vehicle) is to serve the customer needs (maximum power extraction from sun, battery charge based on driving schedules). IT advancement is encouraging investment into smart technologies that can ensure customer needs are met, but, when possible, adjust the timing of energy usage such 
that grid users have lower electricity bills. To accomplish this, the embedded automation has to both ensure the customer needs are served, and satisfy commitments at the grid interface over slower time intervals. These commitments need to be coordinated by an entity through transparent mechanisms. Having suitable protocols is crucial for aligning these two distinct objectives. Shown in Fig. 2 is the representation of how energy-based modeling in [9] can be utilized to map physical and system-level control objectives.

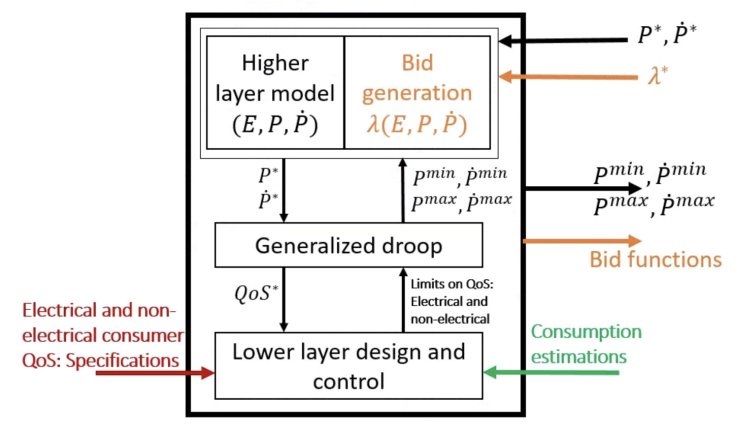

Figure 2: Interactive modeling for control design of DERs

The lower layer comprises the physical model driven by exogenous inputs and grid-side inputs. Here, the device-specific expertise can be utilized towards designing the control to ensure the QoS specifications both at the user interface and grid interface are satisfied [6]. Device interaction with the electrical grid can be captured using a unified energy-space based model that is governed by the dynamics of stored energy and its rate of change. This interaction model is defined using a triplet of energy $(E)$, power $(P)$, and rate of change of power $(\dot{P})$ that is injected into the DER; which can be interpreted as a generalized droop [4]. The intermediate block, labeled as generalized droop can be used to map the higher layer specifications in terms of energy and power to the lower layer QoS specifications. The proposed protocols for the information exchange across the devices is to exchange the triplet comprising stored energy, power and rate of change of power; these physical signals are shown in black in Fig. 2, while the associated cost function is shown in orange. The modeling within the devices is itself interactive. User preferences and learned consumption patterns are utilized to obtain bounds on QoS specifications that are possible to be met given the limits on control and physical variables. These limits along with the cleared prices are utilized to design the predictive controllers to create bid functions over much slower time scales. These bid functions are taken into consideration by the neighborhood system coordinator (compute node in Fig. 3) to then dispatch within the band of allowed power and rate of change of power shown as incoming arrows with quantities $\left(P^{*}, \dot{P}^{*}\right)$. The dispatched quantities are then mapped to the reference points of QoS variables, which are then tracked by the physical device control in the lower layer using fast measurements from local sensors.

The proposed modeling framework offers several advantages: Firstly, the higher layer models utilized in each of the devices are in energy space which is uniform across all energy domains. It offers intuitive engineering appeal for control design, and also is understood by economists for policy making at the system level. Secondly, the grid interactions for both real and reactive power balancing turn out to be linear, thus resulting in a dynamic convex optimization problem with a unique solution to be solved at the system level [9], lending itself to distributed decision making. Thirdly, the issue of multiple local optima leading to non-transparent pricing mechanisms is resolved due to the convexity of the problem in energy space. This leads to perfect alignment of physical and economic signals. Finally, the only information that each device outputs is the bid for real power and its rate of change as shown in Fig. 2. As a result, the device-specific information is hidden, providing an added layer of security. Thus, the proposed interactive modeling resolves the issues of provable device-level control, easy system integration, and also improves security.

The unified interface specification combined with provable devicelevel control facilitate straightforward composition of aggregate models. For instance, several DER models within a household together can be utilized to obtain an aggregate household model (programmable non-intrusive load monitoring devices such as Pecan BluCubes [22]) which can then be utilized to compose neighborhood models. As a result, zooming in/out as desired for the specific layer of interest and the incorporation of corresponding models onto the DyMonDS platform becomes straightforward. The energybased modeling and control embedded in the DyMonDS platform thus offer a holistic approach to systematic integration of DERs and address the technical, economic and cyber concerns in an integrated manner. In Fig. 1, the platform facilitating information exchange between the IoT devices is overlaid on top of the physical grid. The platform can be tailored with additional features that blockchains have to offer, and to have transparent and reliable electricity services, described in the rest of the paper.

\section{SECURE DYMONDS DESIGN}

\subsection{Design Goals}

The aim of the secure blockchain-enabled DyMonDS is to provide functional energy grid management that uses responsive IoT devices to optimize grid functionality, while maintaining a high level of data security and robustness. As part of this design, we explore the role of blockchain as a tool for improving energy systems, subject to the computational and physical constraints of the various system components. This includes considering memory and processing limits of the participants, as well as evaluating the underlying blockchain infrastructure itself, which is typically computationallyintensive to maintain. In this highly inter-connected system, security measures must be in place to protect user privacy and data integrity against potential malicious attacks. Such a system is a valuable addition to the energy space, because as IoT devices become more popular, there arise more opportunities for optimization, but also more potential security concerns.

\subsection{System Overview and Participant Roles}

The secure blockchain-enabled DyMonDS implementation includes two different categories of participants: DERs and compute nodes. This system can be generalized to different devices, but in this particular application, each DER is a BluCube (physical device with embedded intelligence) installed in a Pecan Street household 
[22]; the households are organized in neighborhoods, where each neighborhood has a compute node. The DERs are responsible for sending bids to compute nodes; these bids are generated locally based on device characteristics and previously received systemlevel information, as described in Section 2 and shown in Fig. 2. DERs are treated as relatively trusted sources, but are continuously validated by the compute nodes, discussed further in Section 4 . The compute nodes together act as a centralized management body for the system, so we will consider them trusted (but are still subject to peer-to-peer validation). They are responsible for collecting bids, and using them to generate optimal schedules and LMPs, which are then sent back to the respective DERs. A simpler view of the cyber-

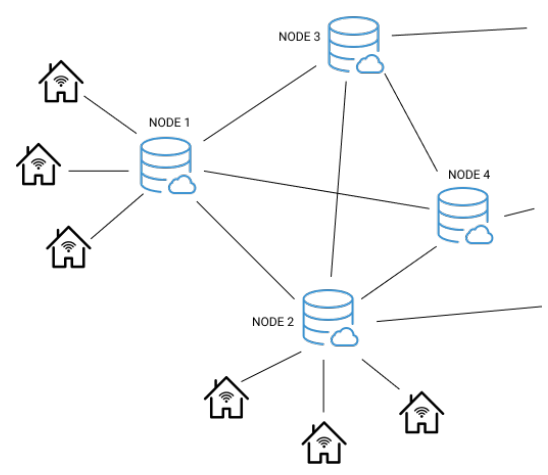

Figure 3: DERs (black) directly communicate with their local neighborhood compute node (blue). Compute nodes are connected to each other via a blockchain network.

physical system in Fig. 1 representing the connections between the two types of participants is shown in Fig. 3. The communication between the DERs and compute nodes is dictated by a secure TCPlike protocol; when a data packet is sent, a record of this interaction is stored, so that if the recipient does not acknowledge that the packet was received, the packet will be resent. Once a compute node had acknowledged the data acquisition, the DER can incrementally clear data from its limited local memory. Introducing a structured communication protocol between DERs and compute nodes enables the system to take advantage of the robustness provided by the blockchain-backed data replication, but also enables low-memory devices to participate in the system.

Compute nodes are connected to each other via a meshed blockchain network, discussed in more detail in Section 3.3. Each of these nodes is therefore responsible for maintaining the distributed ledger of data (bids and optimal solutions), and keeping this ledger synchronized across all nodes. This system assumes collaborative compute nodes that fully exchange all of the information they contain. More specifically, all of the DERs in some neighborhood N1 will send their bids to the N1 compute node. The N1 compute node will use an internal clearing mechanism to find an optimal result for that neighborhood. It will then attempt to write the new N1 data to the blockchain, and broadcast these bids and results to all other compute nodes. Once the data from all compute nodes has been synchronized across devices, the data from each will be permanently written to the blockchain ledger, shown in Fig. 4.

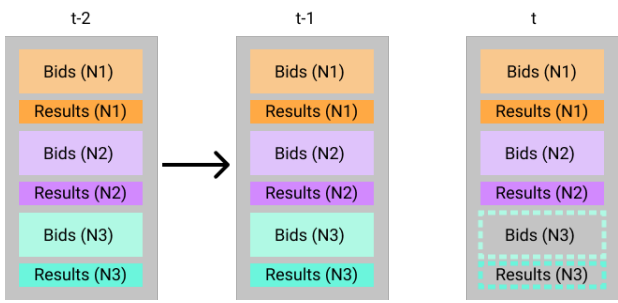

Figure 4: Snapshot of the blockchain ledger that is replicated across all devices. Each "block" in the chain stores data about the bids and optimally computed clearing results. There are three neighborhoods, and all of the results at time $t-2$ and $t-1$ have already been published to the chain. At time $t$, the data from N3 (dotted lines) have been broadcast, but not yet synchronized across all devices. After synchronization, the new block can be appended to the chain (black arrow).

\subsection{Utilizing Blockchain}

3.3.1 Blockchain Background: At its most basic level, blockchain can be defined as a system that uses a peer-to-peer (P2P) network of computers to maintain a ledger of information. Who can write to the ledger, when the ledger can be written, how the ledger is validated, and what is actually being written all depend on the particular implementation. Thus, the question of what would be considered a "blockchain" implementation requires a discussion of privacy, trust, and decision-making power. Some notable features of popular blockchain networks like Bitcoin and Ethereum are that they are public and lack a centralized governing body, yet are both trustless and secure. Trustless blockchains are enabled by some source of scarcity that then makes taking control of the network challenging for any particular - potentially malicious - entity. This scarcity generally enters the system through the consensus mechanism: for Proof of Work (PoW), the limited resource is hashing power; for Proof of Stake (PoS), it is stake in the network. For a blockchain system entirely dedicated to supporting the electric grid, some existing approaches attempt to use energy as that limited resource. However, an obstacle and open question is how to ensure that the energy supposedly produced or consumed is being accurately recorded. PoW solves this problem by using that energy to solve a hash which can then be readily validated, but in a grid management system, energy expended solving a cryptographic puzzle takes away from the value of the system. There ultimately needs to be some verifiable link between the physical IoT devices participating in the system and the data being broadcast about the usage behavior.

A few different approaches can be taken to incorporating this link between physical hardware and broadcasted data. One decentralized option is to allow any device to join, then to use historic data to gauge whether the behavior is plausible for the purported device. However, such a system could be easily skewed by artificially constructing malicious signals. Another approach is to have a fully private chain where every potential participant must undergo a form of validation or screening that then labels them as "trusted", enabling them thereafter to read or write to the chain. In order to make such an assumption in a manner that does not compromise system security, this would involve some centralization to dictate 
which nodes could be labeled as trusted. The restriction that every node must be fully trusted in order to participate can also be relaxed if different nodes have different roles, which is the option selected for the DyMonDS design.

3.3.2 Blockchain Use in the Secure DyMonDS Design: Each of the compute nodes shown in Fig. 3 is a P2P participant in the blockchain network; hence, the links between them are represented with meshed connections. The DERs, which are much more challenging to secure, do not participate directly in the network, and instead communicate with their local compute node.

In order to accomplish the overarching goal of building a system that is designed for energy management, the underlying blockchain that facilitates the interactions between the compute nodes should be energy-conscious as well. Rather than incentivizing network participants to validate data by paying out cryptocurrency (as is done with Bitcoin and Ethereum), the coordinating body is instead fully responsible for validating the system. By limiting participation in this network to the trusted compute nodes, there is no longer a risk of a malicious entity overtaking the network, and thus no need for a PoW-like consensus mechanism. Many possible chains, such as the Energy Web Foundation's Proof of Authority, could be well-suited for this use case, which demands fast network speed, and will take advantage of centralization to perform validation amongst trusted parties [14].

Adding new compute nodes to this system therefore requires going directly through the trusted source responsible for managing the network. The DERs participating in this system do not require the same level of trust since they are also given less responsibility within the network; their role is simply to share bids and respond accordingly. However, onboarding new DERs will still be somewhat regulated in order to link digital device representation and actual hardware. This can be done by interfacing with hardware companies, then requiring users to input some verifiable device serial number or other uniquely identifying specification.

This system design may appear to strip away a big portion of the theoretic value of blockchain systems, because it requires centralization and trust between some nodes. However, looking at this system design in context, we can see that it provides advantages over the grid as it exists today. In today's energy systems, participants are not anonymous, and there is trust in a centralized governing body. While a blockchain system that violates pure trustlessness might seem to take away some of the possible value added, looking at the actual application, blockchain can be used to provide increased security and robust decentralization relative to the existing widely adopted system. Additionally, since reliable IoT energy systems require that there be a provable link between broadcasted data and a physical device, there will always be at some point a layer of trust necessary to verify this connection.

3.3.3 Comparison to a Shared Database: The blockchain portion of this DyMonDS infrastructure essentially operates as a shared database, with some restrictions on which entities have read/write capabilities. A major advantage of a blockchain-backed system is the robustness that is provided due to distributed, local copies of the data. In a traditional database model, data would be stored in a central server, which both introduces vulnerabilities in the case of server failures, and also creates a single point of communication that can serve as a communication bottleneck. Data replication can serve to alleviate some of these issues, but still contrasts with a blockchain system where every participating node has a full ledger of the data. One major disadvantage of the data replication in blockchains is that high communication traffic is needed in order to ensure that the data is synchronized across devices. For DyMonDS, the aforementioned hybrid solution can satisfy the needs and constraints of the system. The DERs lack high memory capability, and thus storing the entire historical ledger is infeasible and unnecessary. The meshed, synchronized blockchain network thus exists only between compute nodes, which are capable of handling this volume of data. A huge benefit of the DyMonDS framework is that individual participants need only be exposed to a limited subset of external variables in order do perform their local computations, reducing network strain and data storage requirements.

\subsection{Blockchain Network Security}

The integrity of the data in the distributed ledger is maintained through the particular chain's consensus mechanism, which ensures that participating nodes agree on what should next be written to the chain before the new data is appended. Additionally, since this ledger is duplicated across all participating nodes, even if an individual node's data becomes corrupted, any alterations will be simple for other nodes to detect. In order to facilitate these distributed practices, the nodes must communicate over a network, which introduces another potential point of vulnerability. However, the distributed nature also protects network security, because even if one communication channel between nodes is compromised, those nodes are also connected to other nodes, so an attacker would have to simultaneously intercept all of these connections. Additionally, it is typical to provide further security by using digital signatures (discussed further in Section 4) and a structured communication protocols to validate participant identity and ensure that data is communicated as intended. However, even if the blockchain portion of the system can be considered secure, the system as a whole still requires additional security mechanisms in order to the protect the more vulnerable components against cyber attack: namely the IoT devices and the communication between them.

\section{SYSTEM SECURITY}

The standard cybersecurity concerns for any connected, computational system are confidentiality, integrity, and availability.For the DyMonDS system design, confidentiality refers to protecting participant privacy leakages [17], and protecting the internal calculations from being exposed. Data integrity is important because the smart meter data is then used by the compute nodes to find an optimal global allocation of energy. Similarly, in order for the devices to have enough time to respond appropriately to the data sent back from the compute nodes, system availability cannot be compromised [26]. Security vulnerabilities exist at all levels of smart grids, but we focus on the connection between household smart devices and compute nodes. The two-tiered security (with some components more trusted than others) described in this section can be generalized to other systems as well. Cybersecurity is crucial to this system because a security breach could result in exposure of personal data, economic loss, hardware damage, or power outages. 
As with any system, the security mechanisms presented in this section cannot protect against every type of attack.

\subsection{Cybersecurity in Energy Grid Management}

Historically, building in "backdoors" to computer systems was a common practice that allowed trusted bodies to edit portions of the system that would otherwise be protected from direct manipulation [23]. However, as energy systems have becoming increasingly interconnected, these types of backdoors expose the system to potential vulnerabilities. Looking at smart embedded energy devices in particular, simply due to hardware and budget limitations, smart devices often have very limited security mechanisms [1, 3]. Additionally, many smart devices come bundled with extra capabilities unnecessary for that particular device, but that introduce more potential points of attack [15]. For these reasons, even smart devices that have undergone some measure of authentication should not be considered fully trusted, since they are particularly vulnerable to attack.

Security policy has not kept up with the changes in the smart grid system. As a result, while building a physical structure requires complying with standard codes, there exists no parallel for building software. The standards introduced in [15] discuss strategies for maintaining the CIA triad, and are considered in the DyMonDS designed. This includes using digital signatures for identity verification, component separation to prevent weaker parts of the system from compromising the whole system, and taking advantage of established cryptographic methods (rather than designing new ones) to protect data integrity.

\subsection{Learning-Enabled Validation}

The data being exchanged in smart grid systems are far from random; physical constraints, known ambient conditions, and behavioral patterns all contribute to determining what "typical" grid interactions will be. Some approaches use machine learning to learn typical behavior, and thereby detect anomalous behavior [12, 13, 27] Others model potential threats with mathematical processes (i.e. as a Gaussian process [2]). Significant work has gone into detecting malicious behavior even when the number of potentially compromised components is unknown [25]. We propose a combination solution that uses both a purely data-driven machine learning approach, but also validates using knowledge about various periodic trends in energy usage shown through decomposition modeling in [7]. While the DyMonDS design takes advantage of relatively trusted compute nodes (defined as "trusted" in the sense that they can write to the blockchain), these will be constantly validated in a P2P manner. Often the physical grid structure is encoded for performing $\mathrm{P} 2 \mathrm{P}$ validation. Consider the three neighborhoods in Fig. 1, the validation of adjacent neighborhoods before they log the new cleared prices to the ledger is validated by knowing that the cleared price has to stay close to its own cleared price at point of interconnection. Any discrepancy indicated that the system has been compromised and thus the new entry does not get logged into any of the other copies of the blockchain ledger. In addition, the compute nodes also hold the software that validates the data exchanged with the DERs.

\subsection{Data Privacy}

With the DyMonDS framework, explicit energy usage data is already protected since those numbers are not being sent directly; bids (which are a function of usage) are sent instead. The internal programs dictating how the IoT devices function and generating bids will never been sent across the network. However, the bids might reveal some information about usage behavior, so even bids should not be readable by external parties. The two primary ways that data might be read by external parties are by viewing the stored data, or by intercepting the data being broadcast between DERs and compute nodes. Both of these vulnerabilities can be addressed by using public-key encryption. The communication between DERs and compute nodes will always be encrypted, so any data sent will be encrypted with the recipient's public key, enabling only the target recipient to decrypt that data using their private key. The stored data will also be encrypted, so that even if an attacker is granted access to the database, no information is exposed unless they also have the necessary private key.

Public-key cryptography is a widely used security mechanism, but requires that the private keys are, in fact, private. If private keys are revealed, then the security breaks. To address this source of vulnerability, the DyMonDS implementation will adopt one of the existing systems that specify policies for key generation, distribution, and management that aim to eliminate those risks for smart devices that would otherwise be likely points of attack [18, 21, 24].

\subsection{DER Data Integrity}

In order for the compute node to verify that the data it is receiving is coming from a valid DER and vice versa, another form of public-key encryption will be used to provide authentication: digital signatures. With a digital signature, each (encrypted) data backed will be "signed" with the sender's private key. When this packet is received, the recipient can use the signer's public key to verify the identity of the sender. Since the DERs are IoT devices that are theoretically accessible from malicious attackers outside of the system, the data they send to the compute nodes could be corrupted. To protect compute nodes against attacks where they receive maliciously planted data (either due to device takeover or entirely fabricated data), the compute nodes will continually validate the incoming data from each of the DERs submitting bids. The compute nodes have knowledge about the type of device broadcasting data, so physically infeasible outputs can be flagged. Additionally, the compute nodes have a full record of the historical broadcasted data from each device, so machine learning can be used to learn trends in behavior. Anomalous behavior can then be detected and flagged. Note that this acts as a detection mechanism, not a prevention mechanism.

\subsection{Malicious Writes}

Even if a malicious attacker is not able to read the encrypted data being sent across the network, they may be able to perform a "manin-the-middle" attack, where they intercept a message, alter it, then forward it along to the intended recipient. This might result in a meaningless decrypted message, in which case, a request could be made to resend the data. However, if they have some knowledge 
about the encryption scheme or data format, they may be able to alter the message in some way that results in a realistic, but corrupted decrypted message. To prevent this type of data tampering, all messages will have a message authentication code (MAC) attached. A MAC is a direct function of the message, so if the underlying data is altered, the MAC will no longer match the message. When the recipient decrypts a message, the MAC enables them to detect if the message has been altered, in which case they can request a resend.

\section{SIMULATION VALIDATION}

We have shown in Section 2 how the proposed DyMonDS-based minimum exchange framework lends itself to secure integration of IoT devices. We will further show that the DyMonDS-based information exchange framework not only abstracts the inner details of IoT devices, but also enables efficient power delivery within the neighborhood. We consider an experiment where neighborhoods shown in Fig. 1 can jointly adjust the overall consumption of the IoT devices within, to manage the uncertainty of the solar power injections. This system is a real-world distribution feeder serving a commercial complex [20]. It consists of small diesel gensets located at buses 22 are 23 with a nameplate capacity of $1 M V A, 4 M V A$; a ramp rate of $20 \mathrm{KW} / \mathrm{min}$ and $8 \mathrm{KW} / \mathrm{min}$ and an operating cost of $10 \$ / M W h$ and $7.5 \$ / M W h$ respectively. The diesel gensets have smart control capable of communicating with the compute node through a bi-directional communication channel. Assumed fast generation from the utility grid is $30 \$ / M W h$. Furthermore, there are three neighborhoods which consist of residential and commercial IoT devices, the respective number of instances of which are further indicated in the figure. We assume that these IoT devices are all HVAC units, with a nameplate capacity varying from $2-12 \mathrm{KW}$ and the temperature settings of the consumers ranging from 72-75 deg F with a temperature variation tolerance ranging from 2-4 deg $\mathrm{F}$ across all the households. In total, the installed HVAC capacity in the grid is about $5.56 \mathrm{MW}$ and the consumption may vary depending on the ambient temperatures which change from $39 \mathrm{degF}$ to $43 \mathrm{degF}$ during the three hours under consideration. Furthermore, the grid has a large solar PV installation injecting high-frequency disturbances as shown in yellow in Fig. 5. This problem is further complicated when accounting for the thermal limits of the lines and the voltage constraints dictating QoS [19]. The intricacies of the complex static optimization are handled by NETSS ACOPF software within the compute node [10].

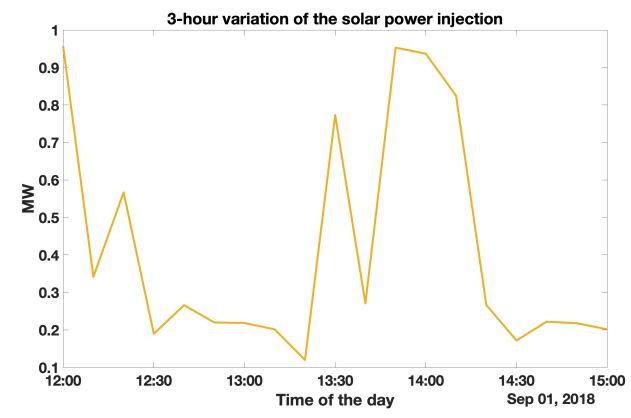

Figure 5: Solar power variations on a typical day
The present operating practice is to balance power in a centralized way, which fails to account for local uncertainties due to prohibitively high memory requirements. We analyze here how local variations can be better taken into account by letting IoT devices compute and communicate the bids as shown in Fig. 2 to the respective compute node as shown in Fig. 1. In this experiment, the IoT devices have enhanced data analytics tools allowing the local consumption-related uncertainties to be predicted ahead of the time accurately. We first let the IoT devices communicate their preferences to the neighborhood operator which in turn log the aggregate bid values into the blockchain ledger, which then get cleared in a P2P way. The clearing mechanism within and across the neighborhood is simulated using a timestep of 10 minutes where the IoT device bids are created by utilizing the algorithms in [11] by looking ahead 2 hours in the future. We compare how the centralized sensing and controlling of the equipment by a single compute node differs from the DyMonDS-based local sensing and distributed control. as depicted in Fig. 1.

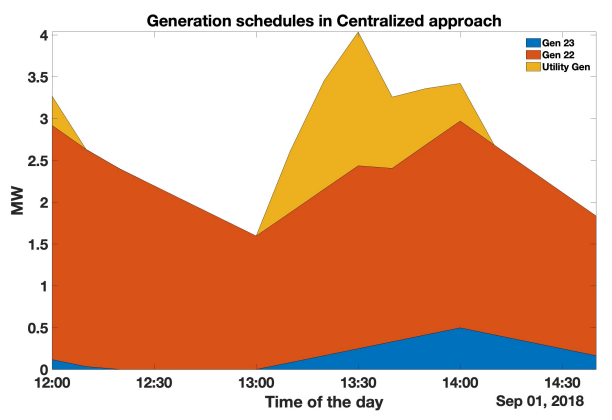

Figure 6: Centralized approach: Increased utility generation and sudden ramping up and down of resources

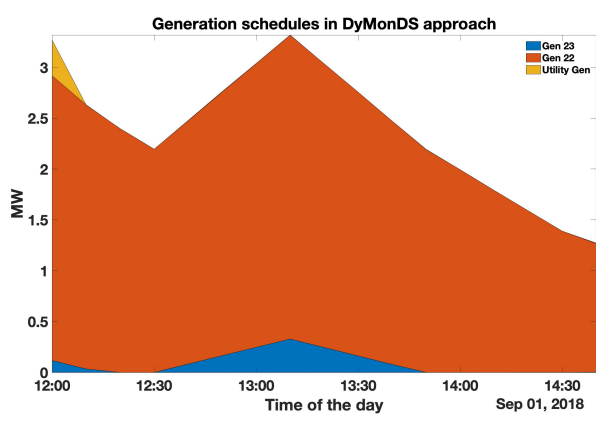

Figure 7: DyMonDS approach: Increased local resource utilization with smooth variations in generation schedules

The generation schedules of the diesel gensets and the utility generators as the neighborhoods adjust their demand is shown in Fig. and respectively for the cases of centralized and distributed clearing. Notice how with centralized clearing, inefficient operation is obtained through increased expensive power imports from the utility grid shown in yellow. On the other hand, looking ahead into 
the future, it has been possible with DyMonNDS-based approach to have efficient utilization of local resources.

A good measure of the overall market efficiency is the value of marginal surplus computed as the difference between the generation revenues and the load charges. Shown in Fig. 8 is the time variation of marginal surplus for every 10-minute time step with the centralized and DyMonDS-based clearing, overlaid on top of each other for comparison. The marginal surplus remains close to zero in the case of DyMonDS-based clearing indicating that the cash flows between producers and consumers are balanced, without having the coordinator to lose or gain. From this experiment,

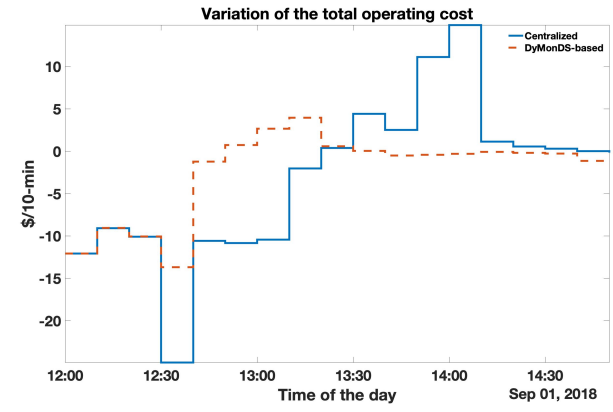

Figure 8: Time variation of the operating cost of the neighborhood

the merits of the DyMonDS-based secure platform design is clear. The proposed design is suitable for scaling to several spatial and temporal hierarchical layers of coordination within the grid, while still resulting in efficient, secure and reliable operation of the grid.

\section{CONCLUSION AND FUTURE WORK}

We have presented a system design that leverages the optimal minimal-exchange DyMonDS framework in order to enable secure communication between between household-level DERs and neighborhood-level compute nodes. Taking the mathematical formulations necessary to provide optimal grid-wide solutions and designing a system around them is a crucial step in order to change and improve grid management today; in doing so, making such systems cyber-secure at the outset rather than as an afterthought will be necessary for being able to support these increasingly interconnected systems. The architecture presented in this paper can be extended to higher, more aggregated levels of the energy grid system, or to other domains entirely. Such an extension would require further work to develop a more formal process for on-boarding new actors in a secure manner.

\section{ACKNOWLEDGMENTS}

This material is in part supported by the Department of Energy under Air Force Contract No. FA8721-05-C-0002 and/or FA8702-15D-0001. Any opinions, findings, conclusions or recommendations expressed in this material are those of the authors and do not necessarily reflect the views of the Department of Energy.

\section{REFERENCES}

[1] Frances M Cleveland. 2008. Cyber security issues for advanced metering infrasttructure (ami). In Power and Energy Society General Meeting-Conversion and Delivery of Electrical Energy in the 21st Century, 2008 IEEE. IEEE, 1-5.

[2] Zubair Md Fadlullah, Mostafa M Fouda, Nei Kato, Xuemin Shen, and Yousuke Nozaki. 2011. An early warning system against malicious activities for smart grid communications. IEEE Network 25, 5 (2011).

[3] David Grochocki, Jun Ho Huh, Robin Berthier, Rakesh Bobba, William H Sanders, Alvaro A Cárdenas, and Jorjeta G Jetcheva. 2012. AMI threats, intrusion detection requirements and deployment recommendations. In Smart Grid Communications (SmartGridComm), 2012 IEEE Third International Conference on. IEEE.

[4] Marija Ilic and Rupamathi Jaddivada. 2019, (Under Review). New Energy Space Modeling for Optimization and Control in Electric Energy Systems. Optimization and Engineering (2019, (Under Review)).

[5] Marija Ilić, Rupamathi Jaddivada, and Xia Miao. 2017. Modeling and analysis methods for assessing stability of microgrids. IFAC-PapersOnLine 50, 1 (2017), 5448-5455.

[6] Marija Ilic, MIAO Xia, and Rupamathi Jaddivada. 2018. Plug-And-Play Reconfigurable Electric Power Microgrids. US Patent App. 15/965,823.

[7] Marija Ilic, Le Xie, and Qixing Liu. 2013. Engineering IT-enabled sustainable electricity services: The tale of two low-cost green azores islands. Vol. 30. Springer Science \& Business Media.

[8] Marija D Ilic. 2011. Dynamic monitoring and decision systems for enabling sustainable energy services. Proc. IEEE 99, 1 (2011), 58-79.

[9] Marija D Ilić and Rupamathi Jaddivada. 2018. Multi-layered interactive energy space modeling for near-optimal electrification of terrestrial, shipboard and aircraft systems. Annual Reviews in Control (2018).

[10] Marija D Ilic, Jeffrey H Lang, Eugene Litvinov, and Xiaochuan Luo. 2006. The critical role of computationally robust AC optimal power flow in reliable and efficient reactive power/voltage dispatch. In Power Systems Conference and Exposition, 2006. PSCE'06. 2006 IEEE PES. IEEE, 689-698.

[11] Rupamathi Jaddivada and Marija Ilić. 2017. A distribution management system for implementing synthetic regulation reserve. In Power Symposium (NAPS), 2017 North American. IEEE, 1-6.

[12] Anish Jindal, Amit Dua, Kuljeet Kaur, Mukesh Singh, Neeraj Kumar, and S Mishra. 2016. Decision tree and SVM-based data analytics for theft detection in smart grid. IEEE Transactions on Industrial Informatics 12, 3 (2016), 1005-1016.

[13] Paria Jokar, Nasim Arianpoo, Victor CM Leung, et al. 2016. Electricity Theft Detection in AMI Using Customers' Consumption Patterns. IEEE Trans. Smart Grid (2016).

[14] Jonas Bentke. 2018. Proof of Authority - EWF - Energy Web Foundation. https://energyweb.atlassian.net/wiki/spaces/EWF/pages/11993089/Proof+ of + Authority

[15] Carl E Landwehr and Alfonso Valdes. 2017. Building Code for Power System Software Security. Technical Report. IEEE Computer Society (2017).

[16] Frank L Lewis and Derong Liu. 2013. Reinforcement learning and approximate dynamic programming for feedback control. Vol. 17. John Wiley \& Sons.

[17] Mikhail A Lisovich, Deirdre K Mulligan, and Stephen B Wicker. 2010. Inferring personal information from demand-response systems. IEEE Security \& Privacy 8, 1 (2010).

[18] Nian Liu, Jinshan Chen, Lin Zhu, Jianhua Zhang, and Yanling He. 2013. A key management scheme for secure communications of advanced metering infrastructure in smart grid. IEEE Transactions on Industrial electronics 60, 10 (2013), 4746-4756.

[19] Chris Punt. 2016. Microgrid Controller Symposium.

[20] RO Salcedo, JK Nowocin, CL Smith, RP Rekha, EG Corbett, ER Limpaecher, and JM LaPenta. 2016. Development of a real-time Hardware-in-the-Loop power systems simulation platform to evaluate commercial microgrid controllers. Technical Report. Massachusetts Inst of Tech Lexington Lincoln lab.

[21] Jia-Lun Tsai and Nai-Wei Lo. 2016. Secure anonymous key distribution scheme for smart grid. IEEE transactions on smart grid 7, 2 (2016), 906-914.

[22] J Vitter and Michael Webber. 2018. Water event categorization using sub-metered water and coincident electricity data. Water 10, 6 (2018), 714.

[23] Paul J Werbos. 2018. AI Intelligence for the Grid 16 Years Later: Progress, Challenges and Lessons for Other Sectors. In 2018 International foint Conference on Neural Networks (IFCNN). IEEE, 1-8.

[24] Jinyue Xia and Yongge Wang. 2012. Secure key distribution for the smart grid. IEEE Transactions on Smart Grid 3, 3 (2012), 1437-1443.

[25] Zhifeng Xiao, Yang Xiao, and David Hung-Chang Du. 2013. Exploring Malicious Meter Inspection in Neighborhood Area Smart Grids. IEEE Trans. Smart Grid (2013).

[26] Ping Yi, Ting Zhu, Qingquan Zhang, Yue Wu, and Jianhua Li. 2014. A denial of service attack in advanced metering infrastructure network. In Communications (ICC), 2014 IEEE International Conference on. IEEE, 1029-1034.

[27] Yichi Zhang, Lingfeng Wang, Weiqing Sun, Robert C Green II, and Mansoor Alam. 2011. Distributed intrusion detection system in a multi-layer network architecture of smart grids. IEEE Trans. Smart Grid 2, 4 (2011), 796-808. 